\title{
ALLERGIC HYPERSENSITIVITY TO FUNGI
}

\author{
By J. PePys, M.B., M.R.C.P., M.R.C.P.E. \\ Senior Lecturer in Allergy, Institute of Diseases of the Chest, Brompton Hospital, London
}

Allergic reactions to the ubiquitous pathogenic and non-pathogenic fungi are models for the study of hypersensitivity. Emphasis will be placed wherever possible in this paper on findings in man, in whom fungal hypersensitivity is widely prevalent. These findings throw light upon fungal antigens in relation to clinical manifestations and upon the nature and mechanism of allergic reactions. It is hoped that this outline will serve as a guide to some of the many problems requiring further study.

A description of certain of the features of the three clearly defined types of allergic hypersensitivity will be given as a source of reference for the subsequent discussion.

\section{Delayed, Tuberculin Type of Hypersensitivity}

This is characteristic of the allergy of infection. The reaction to skin tests develops slowly and is maximal at 48 to 72 hours. The antibody responsible for the reaction appears to be linked to cells of the reticulo-endothelial system and is transported by circulating lymphoid cells to the site of the test reaction. Passive transfer of the delayed type of hypersensitivity can be effected by the injection of these cells, but not the serum, of a sensitive donor into a non-sensitive recipient.

\section{Immediate, Anaphylactic Type, Hyper- sensitivity}

This is characteristic of the clinical allergic disorders such as asthma, hay fever, conjunctivitis and urticaria. The reaction to skin tests is of the urticarial weal and flare type, developing within a few minutes, becoming maximal within 20 to 30 minutes and resolving within $I \frac{1}{2}$ to 2 hours. The non-precipitating antibody present in the serum is capable of sensitizing the skin and smooth muscle and is known as reaginic antibody. Passive transfer of the hypersensitivity is effected by the injection of serum from a sensitive donor into a non-sensitive recipient.

\section{Arthus Type Hypersensitivity}

Arthus reactions are customarily studied in experimental animals. Arthus reactions in man have been suspected because of the similarity of the histological changes in Arthus reactions in animals to those found in vascular reactions in ov man which are regarded as being possibly of allergic origin. The Arthus skin test reaction develops after several hours, that is, later than the immediate type weal reaction, and is maximal within 24 hours, that is, earlier than the delayed type reaction. Arthus hypersensitivity is dependent upon precipitating antibody and can be transferred passively by the injection of serum containing precipitins into a non-sensitive re cipient.

The induction of hypersensitivity is influence by antigenic configurations which can be modifie $\bar{\Phi}$ so as to favour one or other form of hypersensitivity (Benacerraf and Gell, 1959; Gell and Benacerraf, 1959). The various types of hypersensitivity may co-exist and the reaction elicited on testing depends upon the antigenic component employed. In addition, it seems that immediate or Arthus reactions or both may interfere with the development of delayed reactions. There are a number of examples where it would be most instructive to make a clear distinction of the various types of hypersensitivity which may be present at the same time.

\section{Fungal Hypersensitivity}

Fungal hypersensitivity may be classified under two main headings: (I) delayed type hypersensitivity associated with pathogenic fungi, and (2) immediate type hypersensitivity associated with non-pathogenic fungi. Whilst these groups are well defined other forms of hypersensitivity may co-exist.

\section{Hypersensitivity to Pathogenic Fungi}

Infections with these fungi provide classical examples of the allergy of infection. For example, Bloch (1928) described the accelerated response, rapid course and early resolution which appeared on re-infection with a fungus of subjects who had previously been infected. This phenomenon is 
analogous to that described by Koch in tuberculous re-infection. The appearance of delayed type hypersensitivity is closely associated with this accelerated response to infection.

\section{Delayed Type Hypersensitivity}

The passive transfer of delayed hypersensitivity in man to tuberculin and other delayed type antigens by the injection of circulating white blood cells from sensitive subjects has been reported by Lawrence (1956). Similar passive transfer of delayed hypersensitivity in animals had been reported earlier by Chase (1945) and has been repeated by many other workers. More recently Rappoport and others (1959) have reported the passive transfer in man (by the same methods) of delayed type hypersensitivity to a fungal antigen, coccidioidin, thus illustrating a common mechanism in delayed type hypersensitivity to a variety of antigens.

Delayed type hypersensitivity is present in the systemic mycotic infections, and in infections confined to the skin. Skin tests with the appropriate antigens give delayed reactions in histoplasmosis, blastomycosis, sporotrichosis, coccidioidomycosis, moniliasis (candidiasis), and ringworm infections.

In some instances cross-sensitivity has been found, for example in histoplasmosis and blastomycosis, and in coccidioidomycosis and blastomycosis. Although this cross-sensitivity may not be of a high order, it may be important. A pertinent example is provided by Peck (1950), who found that delayed skin test reactions to trichophytin were elicited in 60 per cent. of subjects who gave similar reactions to penicillin and in 33.3 per cent. of those who did not react to penicillin. Delayed skin test reactions to both penicillin and trichophytin were elicited in 9.7 per cent. of treated subjects, and to penicillin alone in 3.2 per cent. This cross-sensitivity is relevant to the allergic reactions which may appear within 24 hours after the administration of penicillin for the first time. Caution is necessary at the present time, however, before attributing this to tinea infection, since penicillin may be ingested unwittingly in foodstuffs.

\section{' Id' Eruptions}

These are naturally occurring manifestations in fungal infections, and are regarded as expressions of delayed type reactions in distant tissues to the fungi or their allergenic products. The 'id' eruptions of tinea infections, and possibly of moniliasis, are attributed to haematogenous distribution of antigen to the skin, and include vesicular, morbilliform, lichenoid, urticarial and other manifestations. Injection of antigens such as trichophytin in skin tests may also excite 'id' eruptions (Sulzberger, 1940).

Erythema nodosum may occur in fungal infections, for example in 5 per cent. of patients with coccidioidomycosis (Kligman and DeLamater, I950), and these subjects may show a high degree of sensitivity to coccidioidin (Wilson, 1957). There is some evidence to suggest that erythema nodosum may be an Arthus type reaction, but decisive evidence in favour of delayed or Arthus hypersensitivity is lacking.

\section{Immediate Type Hypersensitivity}

Immediate weal reactions in skin testing have been reported in some of the systemic mycoses, for example in coccidioidomycosis and blastomycosis. In coccidioidomycosis, Hirsch and Benson (r927) and Smith and others (1948) obtained immediate reactions on skin testing. The latter workers elicited immediate reactions to coccidioidin in persons who failed to give delayed reactions, and generalized urticaria appeared in two subjects within 8 and 18 hours respectively after skin testing. A similar case is reported by Krudy and Hoff (1959), their patient also failing to give a delayed reaction. Some confusion exists in these reports since Smith and others (1948) found, as would be expected, that a control test injection with sterile water also produced wealing. The use of prick tests with suitable antigens and of appropriate non-irritant vehicles for the test materials would overcome any doubts as to the reactions. Prick tests with a carbol saline extract of Candida albicans cells gave immediate weal reactions in about one-quarter of asthmatic patients tested by the author. It is not unlikely that similar reactions may occur in the other mycoses, requiring improved antigens or more careful methods of testing for their demonstration.

Immediate weal reactions to trichophytin in the superficial fungus infections of the skin are not uncommon. Marcussen (1937) has described the successful passive transfer of this hypersensitivity in man by the injection of serum, indicating that reaginic antibody is involved. Sulzberger (1932) has also reported that the injection of trichophytin into sensitive subjects may provoke allergic reactions such as asthma and rhinitis, these manifestations also being associated with the presence of reaginic antibody. Anaphylactic and smooth-muscle sensitization to trichophytin in infected animals was reported by Jadassohn and others (1932, 1937).

In a study of trichophytin skin test reactions in patients infected with Trichophyton rubrum and Trichophyton mentagrophytes, Lewis and Hopper (1948) found that in patients infected with 
T. rubrum 53 per cent. gave immediate reactions only, Io per cent. gave delayed reactions only, and 32 per cent. gave both immediate and delayed reactions, the remaining 5 per cent. being negative on testing. In patients infected with $T$. mentagrophytes the results were quite different, only $\mathbf{I} .5$ per cent. giving immediate reactions only, 72.9 per cent. giving delayed reactions only, 1.5 per cent. giving both types of reaction, and 10.6 per cent. no reactions at all. It would seem, therefore, that the appearance of an immediate skin test reaction has militated against the appearance of a delayed reaction. The patterns of reactivity in the two infections probably arise from differences in the antigenic configurations in the two fungi, leading to the production of predominant immediate type hypersensitivity in the $T$. rubrum infection and to predominant delayed type hypersensitivity in the $T$. mentagrophytes infection.

\section{Arthus Type Sensitivity}

Since fungal precipitins have been reported in the serum of man the possibility that Arthus type hypersensitivity is participating in the pathological manifestations should be considered. The presence of these antibodies provides the immunological basis for this type of reaction.

Precipitins have been reported in systemic mycotic infections, such as blastomycosis, coccidioidomycosis and histoplasmosis. Analysis of the tissue reactions of these infections, and in particular of allergic manifestations such as erythema nodosum, for evidence of Arthus type hypersensitivity is indicated. Hirsch and Benson (1927) reported that the immediate weal reaction to a coccidioidin skin test was followed by a late reaction, coming on 6-12 hours later and maximal at $24-36$ hours, this reaction being suggestive of an Arthus reaction.

Precipitins have also been reported against antigens from fungi responsible for superficial skin infections. Pepys, Riddell and Clayton (1959) have demonstrated precipitins in a high proportion of human sera against $T$. rubrum and T. mentagrophytes. Agar-gel tests were performed by the Ouchterlony method (1953), employing antigens derived from the cell-sap of the fungal mats and from the culture medium filtrates. Common antigens were demonstrated in the two Trichophyton species, which appear also to possess antigens in common with other fungi such as Aspergillus fumigatus, Cladosporium herbarum and Penicillium notatum. The presence of these precipitins makes it necessary to re-examine the delayed skin test reaction to trichophytin for the possible participation of Arthus hypersensitivity in its production.
Hypersensitivity to Non-pathogenic Fungi

Fungal spores have become increasingly recognized as inhalant allergens, responsible for asthma, rhinitis and conjunctivitis and perhaps eczema and urticaria. Blackley, in 1873 , inhaled Penicillium spores which provoked a reaction he did not care to repeat, and Van Leeuwen and others (1925) described fungal spores as a cause of asthma, with emphasis on the Aspergillus species. Many other workers have contributed to this subject, and in more recent times Feinberg (1946) played an $\overrightarrow{0}$ important part in giving fungal spores their $\overrightarrow{\vec{H}}$ appropriate place as ubiquitous, common and $\omega$ potent allergens. A comprehensive discussion on $\bar{D}$ outdoor and indoor fungi is provided by Maunsell (I954).

\section{Immediate Type Hypersensitivity}

Aerobiological studies have helped to define a seasonal incidence for respiratory allergic disorders to several important fungi. In Britain 을 the summer grass pollen season is followed closely by increasing amounts of the spores of Clado- $\square$ sporium and Alternaria in the air, which are at their peak in August and September when they are responsible for troublesome autumnal asthma $\vec{\theta}$ Penicillium spores tend to be present throughow of the year, and the Aspergillus spores are mosfi. evident in the winter months. Among other fungt commonly included in investigations are those causing Dry Rot, various Yeasts and Tricothecium. Most workers in various countries seem to emphasize the above fungi in their reports. Considering the vast numbers of fungal species, it would be surprising if the few examples given here are more than a small proportion of the potential fungal inhalant allergens.

Skin tests by the prick method with suitable extracts of the commoner fungal allergens in sensitive subjects give immediate weal reactions, which are of considerable help in elucidating these somewhat obscure causes of allergic symptoms.

\section{Arthus Type Hypersensitivity}

The Aspergillus genus have been mentioned $\frac{D}{2}$ above as an important and early recognized source of inhalant allergens. The potential pathogen $N$ Aspergillus fumigatus has also been identified as a cause of broncho-pulmonary aspergillosis, in 0 which pulmonary eosinophilia is a feature (Hinson $\mathrm{\omega}$ and others, 1952).

In recent investigations (Pepys and others, 1959) it was found that $A$. fumigatus is present in the sputum of asthmatics more frequently than in other patients. Also present in many asthmatics with the hypersensitive type of pulmonary asper-

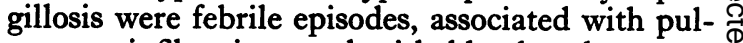
monary infiltrations and with blood and sputum 
eosinophilia, these patients constituting one variety of pulmonary eosinophilia (Crofton and others, 1952).

Skin tests with aspergillus extracts in these patients excited immediate weal reactions and in a number of patients a further reaction appeared several hours after the weal reaction had disappeared. This later reaction was maximal at 24 hours. The immediate weal reactions to skin tests and the asthmatic reaction to inhalation tests indicate that reaginic antibody characteristic of immediate type hypersensitivity was present. The later reaction, however, was suggestive of an Arthus reaction, coming on after the immediate reaction, and being maximal before the delayed reaction. Histological examination of these reactions showed an intense eosinophile cell infiltration and differed from the characteristic perivascular lymphoid cell granuloma of delayed type hypersensitive reactions. Evidence to support the view that Arthus type hypersensitivity was present was provided by the demonstration of Aspergillus precipitins in the serum of many asthmatic patients with $A$. fumigatus in their sputum. These precipitins were demonstrated by the agar-gel diffusion test (Ouchterlony, I953), the antigens being derived from the cell-sap expressed from fungal mats and from the culture medium filtrate.

The presence of common allergens in at least six members of the Aspergillus genus was shown by the immediate weal reactions elicited in most sensitive subjects with extracts of all of the following, $A$. fumigatus, $A$. flavus, $A$. glaucus, $A$. nidulans, $A$. niger, and $A$. terreus. Common antigens in these fungi (except for $A$. glaucus which was not tested) were demonstrated by precipitation reactions in the agar-gel tests with the human sera. Antigens in common with the Aspergillus extracts were also found in the agar-gel tests with extracts of Cladosporium herbarum and Penicillium notatum.

There is evidence, therefore, that both nonprecipitating reaginic antibody and precipitating antibody may be present in patients with the hypersensitive type of pulmonary aspergillosis, and that they may be responsible for different manifestations. Injection treatment with Aspergillus extracts led in some patients to the disappearance of precipitins from the serum, but inhalation tests with Aspergillus extracts in these subjects continued to provoke asthmatic reactions, suggesting that the precipitins were not responsible for the asthma. Precipitins have also been found in non-asthmatic patients with pulmonary Aspergillus mycetomata.

\section{Delayed Type Hypersensitivity \\ Delayed reactions to skin tests with fungal}

allergens are not uncommon, but they have not been investigated sufficiently to indicate whether they are of Arthus or the delayed type. The presence of delayed hypersensitivity cannot be excluded, since immediate weal reactions may interfere with the appearance of delayed skin test reactions.

\section{General Considerations}

The ubiquity of pathogenic and non-pathogenic fungi and of human hypersensitivity to them offers many opportunities for study. The fungi are versatile in the types of hypersensitivity they produce, since the demonstration of human precipitins which may mediate Arthus type reactions to fungal antigens suggests that this type of hypersensitivity in man may be as readily studied as the immediate and delayed types. The high incidence of the various types of antibody in man and the frequency with which exposure to the corresponding fungal antigens occurs poses the interesting question as to whether these may lead to reactions in parts of the body other than those in which the fungi produce their commonly recognized disorders. A recent example of this with other allergens is given in the report by Hardwicke and others (1959) of a case of nephrosis with eosinophil cell infiltration in the kidney, attributed to pollen hypersensitivity.

Analysis of the antigenic patterns of fungi and their further chemical characterization is essential for the provision of the standardized test materials necessary for uniform investigations. For example, the crudeness of the commonly used antigens from Trichophyton and Candida species is a barrier to quantitative measurements of hypersensitivity. In this respect, too, the demonstration of human precipitins to the fungi should be of considerable help.

\section{Localization of ' id' Eruptions}

There are indications from experimental fungal infections of the factors which may influence where and how antigen and antibody may meet in the body, react and set in motion local disturbances. These factors are clearly of wider significance than for fungal hypersensitivity only. The factors which are known to influence the localization of ' $\mathrm{id}$ ' eruptions have some bearing on this problem. Minimal trauma in animals with viable fungi in their circulation leads to localized areas of infection, or in the case of nonviable antigens to the localization of eruptions (Sulzberger, 1940). In man the injection of trichiphytin has been reported to precipitate 'id' eruptions sometimes generalized, though not enough is known to indicate whether these appear at the sites of previous lesions or at the sites of 
trauma. The factors producing local susceptibility of this type may include trauma produced by a variety of agents. To this may be added immunological trauma, such as previous allergic reactions and perhaps also immediate type reactions, which by increasing capillary permeability may permit circulating antigen to meet antibodies in the tissues. Where the antibodies concerned are precipitins more persistent reactions are likely to be excited. In this context it can be postulated that an immediate reaction to one antigen may also permit another antigen to escape from the circulation and to react with antibodies in the tissues. If this hypothesis were sustained, fungi and fungal precipitins might well be prominent causes of such reactions.

Examples of experimental localization of allergic reactions are contained in the report by Seegal and Seegal (1933), that the injection of glycerine into the anterior chamber of the eye of a sensitized animal led to a reaction in that eye, following on the systemic injection of the specific antigen at a later date. When Mott and Kesten (1930) injected whole cells of Candida albicans into the anterior chamber of the eye of animals, followed at a later date by the intravenous injection of a polysaccharide from this fungus, inflammation developed in the previously inoculated eye. It is probable that in these experiments a high local concentration of antibody was produced by the non-specific and specific stimuli, the glycerine and Candida cells respectively. This, together with persistent capillary damage which could encourage local concentration of the antigen as well, may have determined the site of the reaction in the treated eye.

\section{Effect of Immediate Reactions on Appear- ance of Delayed Reactions to Skin Tests}

Whereas it has been suggested above that increased capillary permeability may permit antigen or antibody, or both, to enter the tissues and excite reactions, the position is altered in skin testing in which increased capillary permeability and exudation of serum may inhibit the appearances of reactions at test sites, by diluting and spreading the antigen and by enhancing its absorption.

The possibility that immediate and perhaps Arthus reactions may interfere with the subsequent appearance of a delayed reaction is supported by the report that the production of wealing reactions by a variety of agents of tuberculin test sites leads to the inhibition of the reaction (Pepys, 1955). Allergic weals are equally effective in enhancing absorption of substances from the skin (Abramson and Engel, 1938). The possibility that an immediate or Arthus reaction to an antigen or a member of an antigenic complex may prevent $\frac{2}{2}$ the appearance of a delayed reaction and thereby $\stackrel{\mathbb{Q}}{\subseteq}$ mask the presence of delayed type hypersensitivity $\complement$ must be considered before postulating that other $\overrightarrow{\vec{F}}$ types of antibody are blocking the reaction or $\frac{+}{-}$ that the antigen is altered in some way, thus preventing the delayed reaction. It should be noted $\overline{\bar{N}}$ that $T$. rubrum infection, in which immediate reactions predominate, is more recalcitrant and resistant to treatment than $T$. mentagrophytes os infection, in which delayed reactions predominate. $\overrightarrow{0}$ Similarly, animal ringworm infections give rise $\vec{\overrightarrow{ }}$ to low degrees of delayed hypersensitivity in $\vec{\sigma}$ animals and are refractory in such animals, whereas in man they excite a high degree of $\frac{0}{3}$ delayed hypersensitivity and are more successfully eradicated. In coccidioidomycosis, too, of there are reports (Wilson, 1957) that the presence $\frac{1}{\circ}$ of delayed hypersensitivity is a good, and its absence is a bad, prognostic sign.

These findings suggest that the demonstration 0 of the various types of hypersensitivity may be of $\rightarrow$ importance in assessing host resistance. In cases $\square$ in which one or other of the hypersensitivityc

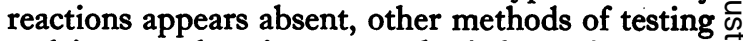
and improved antigens may be informative.

This brief analysis bears out the views of Sulzberger (1940) that familiarity with the allerg. of fungal infections is desirable for the under standing of allergy to infection in general. To this may equally well be added that the understanding of the allergy to fungi is desirable for the understanding of allergic reactions in general.

\section{BIBLIOGRAPHY}

ABRAMSON, H. A., and ENGEL, M. J. (1938), f. invest. Derm., I, 65 .

BENACERRAF, B., and GELL, P. G. H. (r959), Immunology, 2, 53.

BLACKLEY, C. H. (I873), 'Catarrhus Aestivus (Hayfever), its Causes, Treatment, and Effective Prevention,' Experimental Researches, London.

BLOCH, B. (1928), Handb. d. Haut. u. Geschechtskr., II, 300.

CHASE, M. W. (1945), Proc. Soc. exp. Biol. (N.Y.), 59, 134.

CROFTON, J. W., LIVINGSTONE, J. L., OSWALD, N. C., and ROBERTS, A. T. N. (1952), Thorax, 7, 1 .

FEINBERG, S. M. (1946), 'Allergy in Practice,' 2nd ed., The Year Book Publishers Inc., Chicago.

GELL, P. G. H., and BENACERRAF, B. (1959), Immunology, 2, 64. 을

HARDWICKE, J., SOOTHILL, J. F., SQUIRE, J. R., and HOLTI, G. (I959), Lancet, i, 500.

HINSON, K. F. W., MOON, A. J., and PLUMMER, N. S. (1952), 兰 Thorax, 7, 317 .

HIRSCH, E. F., and BENSON, H. (1927), f. infect. Dis., 40, 629. N JADASSOHN, W., SCHAAF, F., and SULZBERGER, M. B. (1932), Klin. Wschr., I1, 857 .

JADASSOHN, W., SCHAAF, F., and WOHLER, G. (1937), Ұ. Immunol., 32, 203.

KLIGMAN, A. M., and Delamater, E. D. (1950), Ann. Rev. O Microbiol., 283.

KRUDY, A. G., and HOFF, R. (1959), Amer. Rev. Tuberc., 79, 78.

LAWRENCE, H. S. (1956), Amer. F. Med., 20, 428.

LEWIS, G. M., and HOPPER, M. F. (r948) 'An Introduction to Medical Mycology,' 3rd ed., The Year Book Publishers Inc., Chicago.

MARCUSSEN, P. V. (1937), Arch. Derm. Syph. (Chicago), 36, 494. 음 MAUNSELL, K. (1954), 'Progress in Allergy,' S. Karger, Basel, p. 457.

Bibliography continued on page 469 
nasal trouble left no doubt about the diagnosis of rhinitis medicamentosa due to adrenergic drugs and in main to naphazoline.

Unfortunately, it is still possible for the patients to obtain these drugs over the counter. Education of the patient is therefore needed, and their co-operation obtained to restrict the use to the worst episodes.

\section{Steroid Hormones}

The second large group of anti-allergic drugs which can exert their action at the site of their deposition on the mucous membrane are steroid hormones. Of the known active adrenocortical compounds, hydrocortisone and prednisolone (deltahydrocortisone) can act locally, whereas cortisone and prednisone cannot. Hydrocortisone is soluble in water, only $\mathrm{I}: 4,000$, and hydrocortisone acetate is insoluble in water. Both drugs can be given as nasal snuff.

Herxheimer and McAllen' (1956) saw good results in hay fever, using $15 \mathrm{mg}$. hydrocortisone locally daily. Good results in nasal allergy with prednisolone locally were reported by Anderson and Ogden (1956). The writer took part in two small controlled trials where patients with hay fever were treated by inhalation of either prednisolone snuff (I mg. twice daily) or by an inert snuff containing lactose (Godfrey, Maunsell, and Pearson, 1957). The results in 1956 suggested that the daily inhalation of $2 \mathrm{mg}$. prednisolone snuff is of considerable value in the control of symptoms due to hay fever. The I956 season was, however, unusually mild, and a further study during the pollen season of 1957 , when pollen counts were much higher, showed that prednisolone snuff alone in dosages of 2-4 mg. did not sufficiently control severe cases. It is, however, considered as a most valuable help in treatment of hay fever and can be given in conjunction with antihistamines and hyposensitization. No side effects were observed; in particular the absence of the rebound phenomenon was noted. Prednisolone as prednisolone sulphate
(Predsol) can be used in watery solution. The drops are instilled three to four times daily and this treatment is of valuable help in controlling seasonal and perennial rhinitis. Polypi sometimes shrink considerably with hydrocortisone snuff as well as with prednisolone. An increase of infected episodes has not been noted.

An important use of prednisolone and hydrocortisone was mentioned previously, i.e. the counteraction of the rebound effect.

Since we have to learn more about the applications and results of the local steroid therapy it seems unfortunate that the pharmaceutical industry set out to combine vasoconstrictors and steroid hormones. For example, Cortibiotic nasal drops contain prednisolone, soframycin and the vasoconstricting phenylephrine; Hydrospray contains hydrocortisone, neomycin and the vasoconstricting propadrin; Efcortelan nasal spray contains hydrocortisone, thiomersal and naphazoline nitrate, and in Delta-Fenox prednisolone is combined with two vasoconstrictors, phenylephrine and naphazoline. Other similar preparations are also on the market.

Since a certain amount of the locally applied hormones will be swallowed after travelling up the nasal passages via the naso-pharynx, one has to watch carefully for toxic general effects. In our cases neither gastro-intestinal trouble nor signs of Cushing's syndrome have been noted. Yet one should be careful and refrain from giving local steroids to patients with gastric ulcers, congestive heart failure, diabetes and tuberculosis. One should not be too enthusiastic about the improvement of the rhinitis and polypi but realize and let the patients realize that these drugs do not cure the disease but only suppress the clinical manifestations.

\section{BIBLIOGRAPHY}

ANDERSON, J. R., and OGDEN, H. D. (1956), Ann. Allergy, I4, 44 .

GODFREY, M. P., MAUNSELL, K., and PEARSON, R. S. Bruce (1957), Lancet, i, 767.

HERXHEIMER, H., and MCALLEN, M. (1956), Ibid., i, 537. LUCAS, H. A. (195I), F. Laryng., 66, 480.

Bibliography continued from page 440-7. Pepys, M.B., M.R.C.P., M.R.C.P.E.

MOTT, E., and KESTEN, H. D. (1930), Proc. Soc. exp. Biol. (N.Y.), 28, 320.

OUCHTERLONY, O. (1953), Acta. path. microbiol. scand., 32, 231. PECK, S. M. (1950), Ann. N.Y. Acad. Sci., 50, 1362.

PEPYS, J. (1955), Amer. Rev. Tuberc. 7x, 40.

PEPYS, J., RIDDELL, R. W., and CLAYTON, Y. M. in press.

PEPYS, J., RIDDELL, R. W., CITRON, K.' M., CLAYTON, Y.'M., and SHORT, E. I., Amer. Rev. Tuberc., forthcoming

RAPPOPORT, F. T., LAWRENCE, H. S., PAPPAGIANIF, D., and SMITH, G. (1959), in press.

SEEGAL, B. C., and SEEGAL, D. J. (1933), F. Immunol., 25, 221.
SMITH, C. E., WHITING, E. G., BAKER, E. C., ROSENBERGER, H. G., BEARD, R. R., and SMITH, M. T. (1948),

SULZBERGER, M. B., and WISE, F. (1932), F. Amer. med. Ass., 99, 1759.

SULZBERGER, M. B. (1940), 'Dermatological Allergy,' C. C. Thomas, Springfield, Illinois.

Van LEEUWEN, W. S., BIEN, Z., KREMER, W., and VAREKAMP, H. (1925), Z. Immun Forsch., 44, $\mathrm{I}$.

WILSON, J. W. (1957), 'Clinical and Immunological Aspects of Fungous Diseases,' C. C. Thomas, Springfield, Illinois.
Bibliography continued from page 466-R. S. Bruce Pearson, D.M., F.R.C.P. QUANLES VAN UFFORD, W. J. (1952), Int. Arch. Allergy

ROSA, L., BERGAMI, G., and CENACCHI, G. C. (1957), Acta. allerg. (Kbh.), 11, 81 .
SAVIDGE, R. S., and BROCKBANK, W. (1954), Lancet, ii, 889. WEST, H. F. (1957), Ann. rheum. Dis., 16, 322.

WEST, H. F. (1958), Ibid., 17, 273.

WILLIAMS, R. S. (1959), Lancet, i, 698. 